\title{
A high-resolution study of mesospheric fine structure with the Jicamarca MST radar
}

\author{
R. Sheth ${ }^{1, *}$, E. Kudeki ${ }^{1}$, G. Lehmacher ${ }^{2}$, M. Sarango ${ }^{3}$, R. Woodman ${ }^{3}$, J. Chau ${ }^{3}$, L. Guo ${ }^{2}$, and P. Reyes ${ }^{3}$ \\ ${ }^{1}$ University of Illinois at Urbana-Champaign, IL 61801, USA \\ ${ }^{2}$ Clemson University, Clemson, SC 29634, USA \\ ${ }^{3}$ Jicamarca Radio Observatory, Lima 13, Peru \\ *now at: Lincoln Laboratories, Lexington, MA 02420, USA
}

Received: 24 August 2005 - Revised: 2 February 2006 - Accepted: 13 March 2006 - Published: 3 July 2006

Part of Special Issue "The 11th International Symposium on Equatorial Aeronomy (ISEA-11), Taipei, May 2005"

\begin{abstract}
Correlation studies performed on data from recent mesospheric experiments conducted with the $50-\mathrm{MHz}$ Jicamarca radar in May 2003 and July 2004 are reported. The study is based on signals detected from a combination of vertical and off-vertical beams. The nominal height resolution was $150 \mathrm{~m}$ and spectral estimates were obtained after $\sim 1 \mathrm{~min}$ integration. Spectral widths and backscattered power generally show positive correlations at upper mesospheric heights in agreement with earlier findings (e.g., Fukao et al., 1980) that upper mesospheric echoes are dominated by isotropic Bragg scatter. In many instances in the upper mesosphere, a weakening of positive correlation away from layer centers (towards top and bottom boundaries) was observed with the aid of improved height resolution. This finding supports the idea that layer edges are dominated by anisotropic turbulence. The data also suggests that negative correlations observed at lower mesospheric heights are caused by scattering from anisotropic structures rather than reflections from sharp vertical gradients in electron density.
\end{abstract}

Keywords. Meteorology and atmospheric dynamics (Turbulence; Middle atmosphere dynamics) - Radio science (Radio wave propagation)

\section{Introduction}

VHF radar returns from the daytime mesosphere are a consequence of electron density variations $\delta N(\boldsymbol{r}, t)$ in the region. When $\delta N(\boldsymbol{r}, t)$ variations are exposed to transmitted radar pulses $\boldsymbol{E}_{i}(\boldsymbol{r})$, oscillating currents $\propto \delta N(\boldsymbol{r}, t) \boldsymbol{E}_{i}(\boldsymbol{r})$ are established. The currents re-radiate electric fields

$\delta \boldsymbol{E}=-r_{e} \int d \boldsymbol{r} \delta N(\boldsymbol{r}, t) \boldsymbol{E}_{i}(\boldsymbol{r}) \frac{e^{-j k_{o} r}}{r}$

Correspondence to: E. Kudeki

(erhan@uiuc.edu) back to the radar antenna. In Eq. (1), $r_{e}$ denotes the classical electron radius, $k_{o}$ is the wavenumber of transmitted and reradiated (or scattered) fields $\boldsymbol{E}_{i}(\boldsymbol{r})$ and $\delta \boldsymbol{E}$, and $r \equiv|\boldsymbol{r}|$ is the radar range. If the variations $\delta N(\boldsymbol{r}, t)$ are random and have a space-time spectrum $\left\langle|\delta N(\omega, \boldsymbol{k})|^{2}\right\rangle$, then the average power delivered by Eq. (1) to the radar receiver can be represented as

$$
\langle P\rangle=\int d r d \Omega \frac{d \omega}{2 \pi} G(\hat{r}) \frac{P_{t}\left(t-\frac{2 r}{c}\right)}{(4 \pi r)^{2}} A(\hat{r}) \sigma_{v},
$$

where $\hat{r} \equiv \boldsymbol{r} / r$ is the radial unit vector pointing away from the radar antenna, $G(\hat{r})=k_{o}^{2} A(\hat{r}) / \pi$ the antenna gain, $P_{t}(t)$ the transmitted pulse power, and

$\sigma_{v} \equiv 4 \pi r_{e}^{2}\left\langle\left|\delta N\left(\omega,-2 k_{o} \hat{r}\right)\right|^{2}\right\rangle$

the backscatter cross-section of $\delta N$ variations per unit volume per Doppler frequency $\frac{\omega}{2 \pi}$.

Since the mesosphere is a weakly ionized and highly collisional gas, electron density variations in the region are expected to be controlled by neutral dynamics. Consequently, when neutral flow is turbulent, the assumptions underlying the backscattered power model (2) should be valid. In regions of laminar flow, on the other hand, stable $\delta N$ gradients capable of causing partial reflected radar pulses can presumably be sustained under the right conditions.

The roles of randomly scattered versus partial reflected fields - both described by Eq. (1) - have been debated since the early days of the MST radar technique (e.g., Woodman and Chu, 1989; Hocking and Röttger, 2001). Since partial reflections are caused by time-invariant density structures their "correlation times" should be long and their Doppler frequency spectra narrow. By contrast turbulent scatter is associated with fast varying $\delta N$ and broad Doppler spectra. In fact, the larger the random velocities in a turbulent flow, the broader the Doppler spectrum and larger the backscattered power since larger random velocities cause more mixing and larger $\delta N$ amplitudes (assuming mixable gradients $d N / d z$ of

Published by Copernicus GmbH on behalf of the European Geosciences Union. 
the background density variation $N(z)$ exist). Thus a positive correlation between radar signal power and spectral width is considered a signature of turbulent backscatter. Conversely, narrow spectra associated with large power returns argue for the dominance of reflections over turbulent scatter.

The first extensive correlation studies of mesospheric echo power and spectral widths were conducted by Fukao et al. (1980). The measurements were taken with the $50 \mathrm{MHz} \mathrm{Ji}-$ camarca MST radar near Lima, Peru, using a height resolution of about $2.5 \mathrm{~km}$. The study revealed positive correlations in the higher mesosphere above $\sim 75 \mathrm{~km}$ implying the dominance of turbulent scattering. Negative correlations were reported for the lower mesosphere, suggestive of an increasing role for reflections at heights closer to the stratosphere, or the possibility of inertial range turbulence as suggested by Røyrvik (1985). Subsequent Jicamarca measurements conducted with a $2 \mathrm{~km}$ resolution (Røyrvik, 1983) showed similar and consistent results, including predominantly "aspect sensitive" returns from below $75 \mathrm{~km}$.

Aspect sensitivity is the dependence of echo power on the direction of radar beam, namely the angular pattern of incident field intensity $\left|\boldsymbol{E}_{i}(\boldsymbol{r})\right|$. Buoyancy effects dictate that in a stable atmosphere with laminar flow $\delta N$ varies only with height $z$. As a consequence Eq. (1) requires a $z$ directed radar beam to produce a partial reflected signal of a substantial amplitude. By contrast the beam direction is inconsequential when $\delta N$ variations are caused by isotropic turbulence. In that case backscatter cross-section $\propto\left\langle\left|\delta N\left(\omega,-2 k_{o} \hat{r}\right)\right|^{2}\right\rangle$ is independent of $\hat{r}$ and radar response is not aspect sensitive. In between the extreme cases of "specular" or aspect-sensitive partial reflections and isotropic backscatter lies the domain of aspect-sensitive backscatter from anisotropic turbulence.

It has been argued by Bolgiano (1968) and later by Woodman and Chu (1989) and Hocking and Röttger (2001) that the boundaries or edges of turbulent layers in the atmosphere should exhibit anisotropic flows - i.e., eddies elongated along the edges - and contain elongated density structures with a spectrum $\left\langle|\delta N(\omega, \boldsymbol{k})|^{2}\right\rangle$ which is sensitive to the direction of wavevector $\boldsymbol{k}$. Radar echoes from turbulent layer edges should then exhibit random statistics as well as aspect sensitivity. The early MST experiments of Fukao et al. (1980) and Røyrvik (1983) lacked the height resolution required to observe the peculiar characteristics of layer boundaries predicted by Bolgiano (1968) and, in general, to distinguish between specular reflections and anisotropic turbulence; the radar volumes often contained a multitude of density structures, potentially associated with different echoing mechanisms.

High resolution radar measurements of mesospheric returns have been conducted since then at Jicamarca and elsewhere. Some of the highest resolution results include direct evidence for backscatter from layer edges (e.g., Reid, 1990) as well as direct observations of Kelvin-Helmholtz billows in radar backscatter maps (e.g., Czechowsky and Rüster, 1997). However, the best examples are usually representative of ex- treme or special conditions - solar flares, unusually strong turbulence, polar-mesosphere summer echoes, etc. - and a broad survey of the morphology of average conditions of mesospheric fine structure has not been reported, at least not from Jicamarca.

By virtue of its superior power-aperture product, the MST radar at Jicamarca is capable of remote sensing the mesosphere over a wider dynamic range than any other existing MST radar at the highest resolution. Expressed in another way, Jicamarca is not constrained to observe the mesospheric fine structure under only extreme or extremely disturbed turbulent or not - conditions.

The present paper describes a Jicamarca based highresolution study of mesospheric radar returns conducted under randomly selected and average geophysical conditions at low-latitudes. The study is essentially an update of the earlier work of Fukao et al. (1980) and Røyrvik (1983) carried out with a nominal height resolution of $150 \mathrm{~m}$ - a standard for state-of-the-art MST radars - and aims a better understanding of mesospheric density structures and related radar scatter at low-latitudes. It is in fact the first correlation study conducted at Jicamarca that "resolves" the echoing layers with many independent range gates.

In Sect. 2 we describe the high resolution radar modes used at Jicamarca to collect the data analyzed in this paper. The section also describes the spectral analysis and fitting procedures used to obtain estimates of the backscattered echo power and Doppler spectral widths. Radar observations and correlation study results are presented in Sect. 3 and discussed in Sect. 4. Sect. 5 summarizes our findings.

\section{Data acquisition and spectral moment estimation}

Jicamarca radar data analyzed in this paper were collected during three days in May 2003 and two days in July 2004. The parameters for both sets of observations are given in Table 1. In May 2003 observations four radar beams pointed $\sim 2.5^{\circ}$ off-vertical in north, south, east, and west directions were used. Two-way patterns of the beams are depicted in Fig. 1a as a function of direction cosines defined in the caption. The beams were excited with 64 baud complementary coded pulse pairs. The interpulse period (IPP) was $1.33 \mathrm{~ms}$ and a baud length of $1 \mu \mathrm{s}$ was used. Matched filtered receiver outputs had a nominal height resolution of $150 \mathrm{~m}$ corresponding to $1 \mu \mathrm{s}$ baud length but were oversampled by a factor of four at $37.5 \mathrm{~m}$ range intervals. The actual height resolution was closer to $250 \mathrm{~m}$ than $150 \mathrm{~m}$ because of imperfections in transmitting and receiving systems. Also, only two of the four beam outputs were sampled after each pulse transmission making the effective IPP $2.66 \mathrm{~ms}$ per beam.

In July 2004 the measurements were conducted using only two beams. One beam was vertical - i.e., directed towards the true zenith - while the second one was in "on-axis" direction normal to the plane of the Jicamarca antenna array, 
Table 1. Experiment parameters used at Jicamarca in May 2003 and July 2004. Range and gap parameters are in $\mathrm{km}$ units and the fifth column gives the number of sampled range gates. The "gap" refers to upper stratospheric heights from which no detectable radio returns are expected. The average spectrum from these heights is regarded as the estimate of the background noise spectrum and is referred to in the text as $B(\omega)$.

\begin{tabular}{cclllc}
\hline Day & LT & Beams & Range & Gates & Gap \\
\hline 27/05/03 & $06: 04-17: 48$ & EWSN & $60-95$ & 920 & $60-63$ \\
28/05/03 & $06: 13-12: 08$ & EWSN & $60-95$ & 920 & $60-63$ \\
28/05/03 & $12: 09-15: 42$ & EWSN & $9.6-94$ & 2260 & $40-50$ \\
$29 / 05 / 03$ & $06: 06-17: 30$ & EWSN & $9.6-94$ & 2260 & $40-50$ \\
$13 / 07 / 04$ & $09: 17-17: 45$ & SW,V & $9.6-94$ & 2260 & $40-50$ \\
$14 / 07 / 04$ & $08: 28-17: 39$ & SW,V & $9.6-94$ & 2260 & $40-50$ \\
\hline
\end{tabular}

about $2.29^{\circ}$ to $\mathrm{SW}$ of the zenith direction. Two-way patterns of July 2004 beams are shown in Fig. 1b. The on-axis beam was obtained with equal phasing and illumination of all array elements and therefore it has the highest aperture efficiency (nominally 100\%) of all the beams shown in Fig. 1. The effective IPP was also $2.66 \mathrm{~ms}$ per beam in July 2004 since the vertical beam returns were sampled three times using the $\mathrm{N}$, $\mathrm{E}$, and $\mathrm{W}$ quarters of the antenna array for purposes of interferometric analysis.

Raw radar data collected in May 2003 were coherently integrated and decoded over blocks of 60 samples corresponding to a post-integration IPP of $0.16 \mathrm{~s}$. 64-point periodograms computed with post-integration samples every $10.24 \mathrm{~s}$ were summed incoherently to obtain Doppler spectral estimates, one per range gate per minute. July 2004 data were coherently integrated twice as long and as a consequence half as many periodograms were summed to obtain spectral estimates per minute.

Doppler spectral estimates obtained during the experiments - to be referred to as measured spectra from hereon - represent the sum of a backscattered signal component and broadband noise. Estimates of the noise component were obtained from "gap" heights - see Table 1 - in the upper stratosphere where backscatter cross-section is vanishingly small. Noise table estimates - which, at times, also includes man made interference - are used in power map formation, model fitting, and the estimation of signal correlation times.

Figure 2 shows a 64-point measured Doppler spectrum example together with several different model fits and horizontal bars representing different estimates of the corresponding spectral width. The area between the measured spectrum curve $S(\omega)$, and a curve $B(\omega)$, representing the background noise deduced from gap heights (not shown in the figure), is the estimate of the backscattered signal power. The first (a)
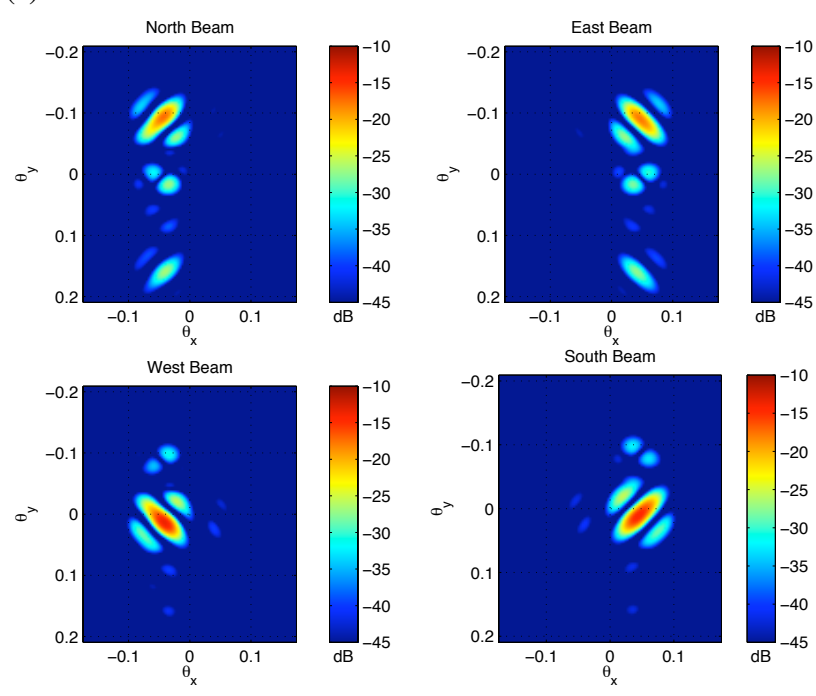

(b)
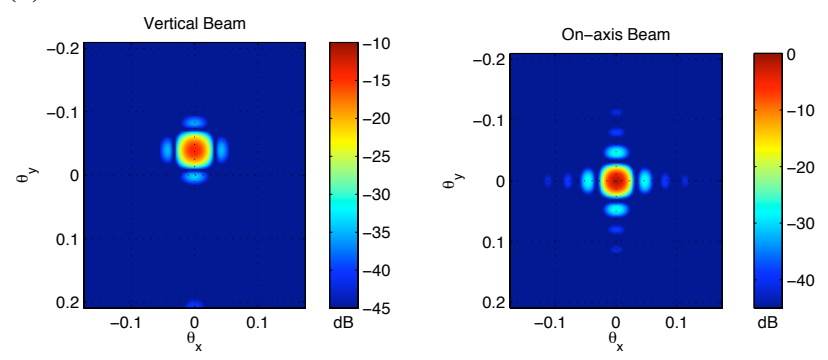

Fig. 1. Two-way antenna beam patterns $\propto G(\hat{r}) A(\hat{r})$ used in (a) May 2004 and (b) in July 2004 experiments. The patterns are shown in $\mathrm{dB}$ with a common normalization and vary with direction cosines $\theta_{x} \equiv \sin \theta \cos \phi$ and $\theta_{y} \equiv \sin \theta \sin \phi$, where $\theta$ is measured from the Jicamarca on-axis position and $\phi$ is azimuth angle measured from SE towards NE. True zenith is in $\theta_{x}=0$ and $\theta_{y}=0.04$ direction, the direction of peak response in the left panel in (b).

and second central moments of the difference $S(\omega)-B(\omega)$, on the other hand, define the Doppler shifts and widths of backscattered radar returns. However, a direct estimation of these moments from the noisy spectrum $S(\omega)$ is often problematic and can lead to - especially in low SNR cases - biased estimates (e.g., Yamamoto et al., 1988). A more robust approach is to calculate the moments from smooth model curves fitted to the measured spectra.

Since no first-principle model exists for the spectrum of mesospheric radar returns, any smooth curve that fits the data well can be used. We have found that generalized-Gaussian based models like

$$
\langle S(\omega)\rangle=A \exp \left\{-\left|\frac{\omega-\mu}{\sigma_{p}}\right|^{p}\right\}+B(\omega)
$$

or

$$
\langle S(\omega)\rangle=B(\omega) \exp \left\{A e^{-\left|\frac{\omega-\mu}{\sigma_{p}}\right|^{p}}\right\},
$$






Fig. 2. Example spectrum (blue) overlaid with yellow, green, and red symbols corresponding to three different fitting procedures described in the text. Spectral width estimates obtained with six different methods are shown as horizontal lines (with matching colors to the diamonds). The green and red data points indicate spectral widths obtained from logarithmic fits applied with spectral models (4) and (5) result in comparable widths shown in green and red, respectively. Spectral moment calculation (blue) and linear generalized Gaussian fit (yellow) give slightly wider estimates. Estimates obtained from the correlation times, with (black) and without (cyan) accounting for triangular weights are the largest for this spectrum.

where $B(\omega)$ is the background noise estimate from the gap, provide smaller misfits than models based on a simple Gaussian. We also found that fitting the natural logarithm of the measured spectrum to the natural logarithm of these models leads to faster convergence and at times smaller misfits than direct fitting.

The spectral model (4) given above reduces to a proper Gaussian for the special case of $p=2$. The fact that the best fits often result in $p$ other than 2 is an indication that mesospheric spectral shapes are often non-Gaussian. This result is consistent with recent studies (e.g., Bahcivan et al., 2003) which point out that MST Doppler spectra can deviate from Gaussian shapes. The second model equation (5) is not related to Gaussian in a straightforward way but it performs even better than the first model in many cases. In both models $\mu$ is the first moment or Doppler spectral shift, whereas the second central moment must be computed numerically from $\langle S(\omega)\rangle-B(\omega)$. While the two models above lead to substantially different fits for $A, p$, and $\sigma_{p}$, spectral width estimates obtained from them usually agree very closely with one another as shown in Fig. 2.

In our spectral fitting we used a misfit definition $\chi^{2}=\sum_{q} \sigma_{q}^{-2}\left(S_{q}-\left\langle S_{q}\right\rangle\right)^{2}$, where $S_{q} \equiv S(q \delta \omega)$, and
$\sigma_{q}=K^{-1 / 2}\left\langle S_{q}\right\rangle$ is the rms error in spectral samples $S_{q}$ obtained by averaging $K$ independent periodograms (e.g., Kudeki et al., 1999). In logarithmic fitting we used $\chi^{2}=K \Sigma_{q}\left(\ln S_{q}-\ln \left\langle S_{q}\right\rangle\right)^{2}$. These two $\chi^{2}$ definitions are mathematically identical in large $K$ limit, but for small $K$ different $\chi^{2}$ values can be expected for the same input parameters $A, p, \sigma_{p}$, and $\mu$. It was found that logarithmic fits work better for very narrow spectra. The difference between the two fitting procedures can be attributed to the fact that the logarithm function changes the minimization space so that a "path" to a minimum in one space may be found that would not exist in the other space. In fact, using the best-fit parameters from logarithmic fit as initial guess to direct fitting will produce a better direct fit than would be obtained using fixed initial conditions.

We used MATLAB's LSQCURVEFIT function with medium-scale operation (Levenberg-Marquardt implementation) for $\chi^{2}$ minimization; stopping criteria was defined as either reaching a maximum number of iterations or having the improvement in taking the next step falling below a certain threshold. It was usually the latter stopping criterion that was reached in most cases.

Figure 2 shows spectral width estimates obtained also as the inverse of signal correlation time derived from backscattered signal ACF. The latter is estimated using the inverse Fourier transform of zero-padded Doppler spectra (with the noise table subtracted) and signal correlation time is defined as the time to 0.5 normalized ACF. The ACF estimate obtained as just described is triangular weighted across time lags and leads to the width estimate shown in black. A second ACF estimate is obtained by dividing the first estimate by the triangular weights and leads to the width estimate shown in cyan. We have found that all spectral width estimates illustrated in Fig. 2 agree very closely in all cases with $\mathrm{SNR}>-5 \mathrm{~dB}$, giving us confidence in our characterization of spectral widths by any one of the methods discussed above.

In this study we examine the correlation between observed spectral widths and returned signal power in order to distinguish between isotropic and anisotropic scatterers. For the purposes of illustration, an example of positive and negative correlations between spectral widths and signal power is shown in Fig. 3. These are scatter plots of spectral widths and SNR in $\mathrm{dB}$ at a single altitude over some time interval. Only measurements with SNR larger than $-5 \mathrm{~dB}$ are used to keep low power returns from biasing the unweighted computation of the correlation coefficient. With low SNR, there is the danger of mistaking the width of the noise table as signal spectral width and infer erroneously negative correlations between SNR and spectral width. This effect can be mitigated further by employing another threshold that checks the number of points available to form a correlation. If this value is below a certain minimum then there is no need to form a correlation since it can be assumed that the linear fit would be biased by noise. 

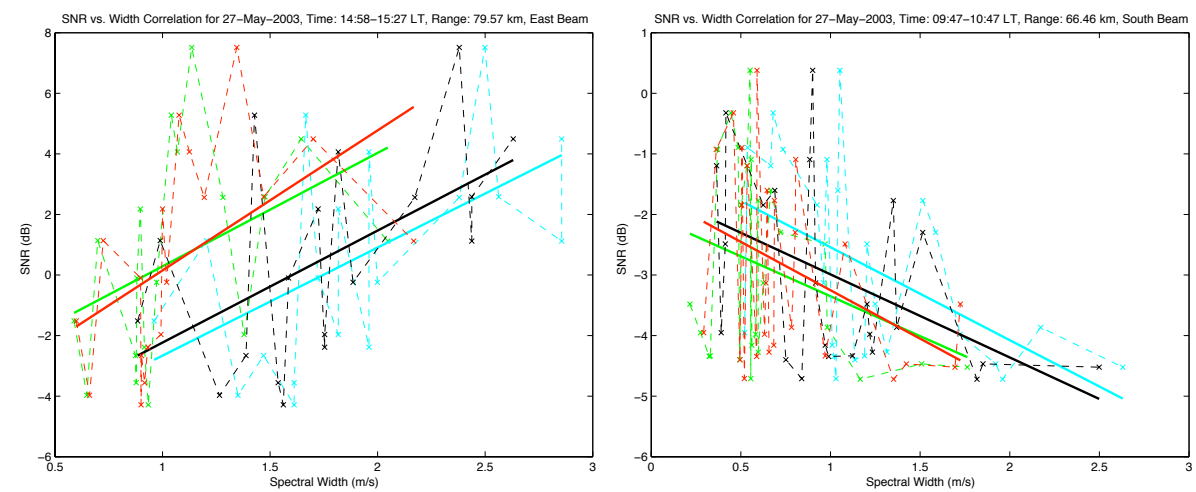

Fig. 3. Examples of positive and negative correlation between SNR and spectral width. The green and red data points indicate spectral widths obtained from logarithmic fitting of spectral models (4) and (5), respectively. The cyan data points are spectral widths obtained from the correlation times, and the black data points are widths obtained from the correlation times with the triangular weighting function taken into account. The solid lines indicate linear fits to their associated data points.

\section{Experimental results}

Backscatter SNR maps for the five days in May 2003 and June 2004 are shown in Fig. 4. Mesospheric echoes depicted in the maps show a variety of behaviors ranging from large turbulent-like structures that last virtually the entire day to collections of smaller "blob"-like features that last less than an hour. The returns from 27-28 May 2003 show broad layered structures above $65 \mathrm{~km}$ with little return below. 29 May 2003 consists of a collection of thin sheets and blobs. The two days from July 2004 both show large turbulent structures above $70 \mathrm{~km}$ and a collection of thin sheets below. Spike-like echoes above $80 \mathrm{~km}$ are due to meteor trails.

To study the height dependence of spectral width vs. SNR, Fukao et al. (1980) used correlations formed over the entire data set. The trend observed was a negative correlation at lower mesospheric heights that gradually became positive with increasing altitude above about $75 \mathrm{~km}$. For purposes of comparison with these earlier observations, correlation plots were formed for 27 and 29 May 2003, which are shown in Fig. 4 next to the SNR maps. A $-5 \mathrm{~dB}$ SNR threshold was used with 10 being the minimum number of points used to form a correlation, and red (cyan) indicates that spectral widths used to calculate the correlations were obtained with fitting (ACF) method.

The correlation plot for 27 May shows mainly positive correlation with well defined peaks at 70 and $73 \mathrm{~km}$, and variable positive correlation above $76 \mathrm{~km}$. The correlation below $70 \mathrm{~km}$ shows mostly negative values and a narrow peak at $67 \mathrm{~km}$. The correlation plot for 29 May illustrates the difficulties in attempting a correlation study over an entire day. Many of the layers observed on this day are descending or ascending, and as a result, the correlation estimates obtained over the whole day are noisy (notice an almost random distribution of positive and negative correlations over height) and difficult to interpret. Clearly, given that the temporal and spatial resolution of the current data allows us to observe individual layer dynamics, correlations should be formed and studied on an event-per-event basis and not over an entire day of observations.

A sequence of SNR maps and correlation coefficient profiles shown starting in Fig. 5 give a representative summary of the individual scattering events and layers which were observed in 27-29 May 2003, and 13-14 July 2004. The correlation estimates for these were once again obtained using a threshold of $-5 \mathrm{~dB}$ SNR and a 10 data point minimum.

Figures 5-6 depict echoing structures from 27 May 2003. On this day a strong turbulent layer was observed at $74 \mathrm{~km}$, located above a thinner layer starting at $70 \mathrm{~km}$ and descending throughout the day as shown in Fig. 4a. Figure 5a shows the correlation plot for a section of the $74 \mathrm{~km}$ layer starting shortly after 10:00 LT. The event shows strong positive correlation at its center with decreasing positive correlation towards the edges. At the very edges there is a slight reversal to negative correlation at both the top and the bottom. Figures $5 \mathrm{~b}-\mathrm{d}$ show further snapshots in the evolution of the $74 \mathrm{~km}$ layer throughout the day. Figure $5 \mathrm{~b}$ shows a positive correlation minimum at the layer center with two peaks of positive correlation above and below. The edges of the layer again show a slight negative correlation. The double-peaked correlation plot is evidence for the existence of two sublayers within the larger turbulence structure. Figure $5 \mathrm{c}$ shows the larger turbulent layer three and a half hours later splitting into two distinct layers. The lower layer still keeps its structure while the upper layer diffuses somewhat. This is evident through the correlation plot by a well-defined peak at $72.5 \mathrm{~km}$ and a smaller, less-defined peak at $75.5 \mathrm{~km}$. The correlation between roughly $74 \mathrm{~km}$ and $75 \mathrm{~km}$ is, on the average, zero with one narrow peak at $75.25 \mathrm{~km}$. It should be noted that spectral width is implicitly assumed to be the independent 

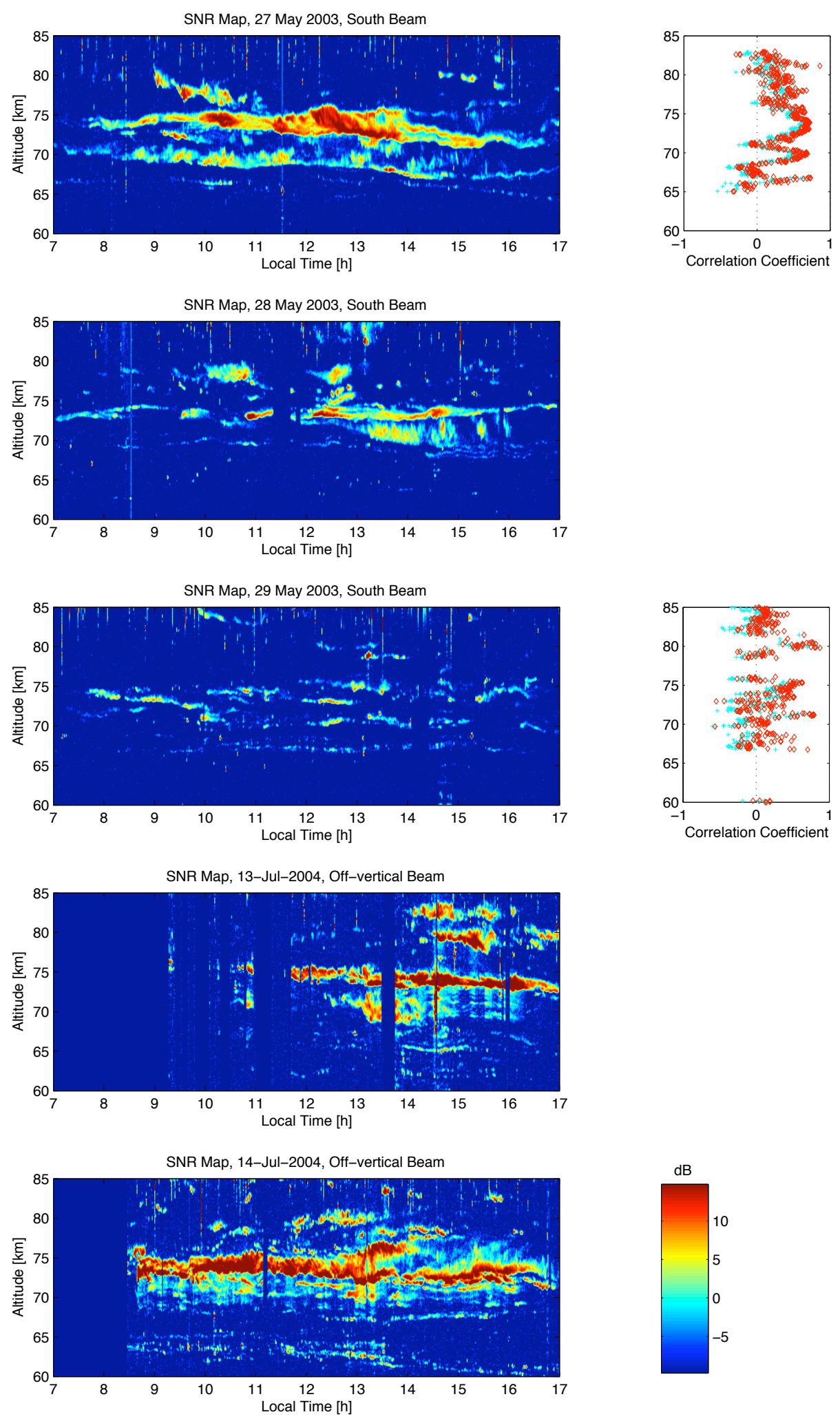

Fig. 4. SNR maps from May 2003 (south beam data) and July 2004 (off-vertical beam) observations. Height profiles shown to the right of top and middle panels correspond to spectral width versus echo power correlation coefficients. 
(a)
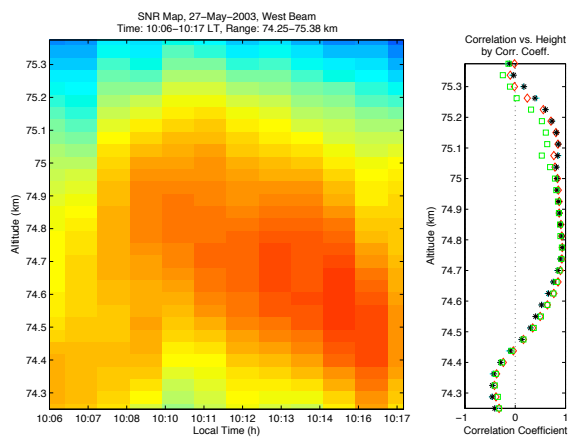

(b)


(c)



(d)
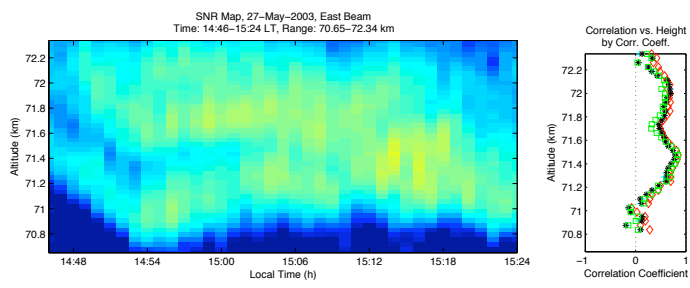

Fig. 5. SNR maps and correlation plots of sections of the turbulent layer at $74 \mathrm{~km}$ on 27 May at (a) 09:54 LT, (b) 12:08 LT, (c) 13:30 LT, and (d) 14:50 LT.

variable in correlation calculations - thus, zero correlation should be interpreted as the following: when spectral width increases, there is no tendency for the backscattered power to either increase or decrease. Figure $5 \mathrm{~d}$ shows the large turbulent layer at the end of the day. It has descended in height and the two sub-layers stand out more clearly. The corre- (a)
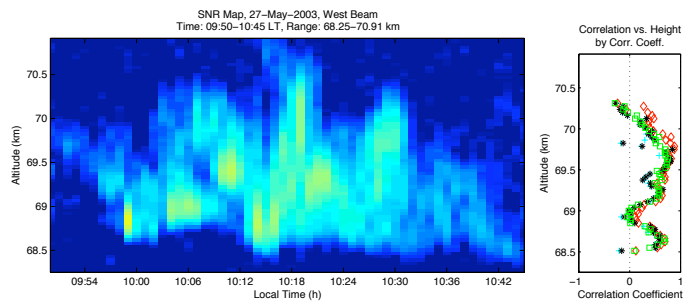

(b)

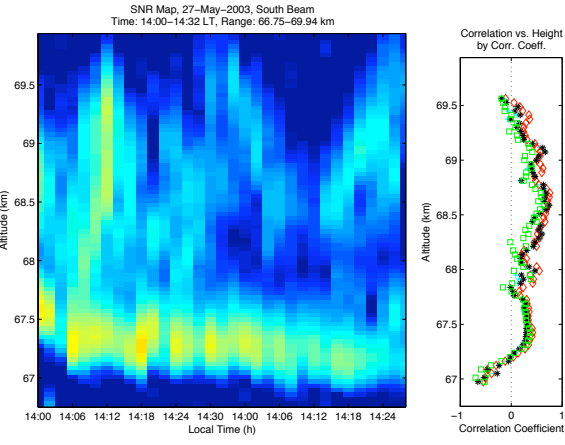

Fig. 6. Thin layer on 27 May (a) at $69 \mathrm{~km}, 13: 00 \mathrm{LT}$ and (a) at $67.4 \mathrm{~km}, 14: 00 \mathrm{LT}$.

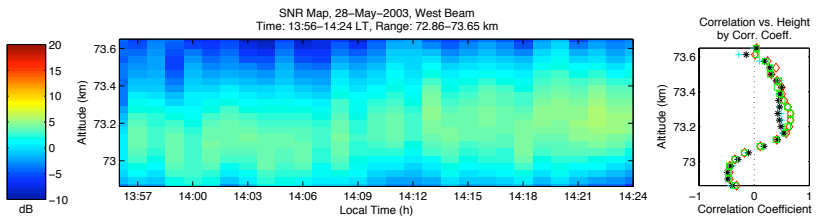

Fig. 7. Turbulent layer centered about $73.2 \mathrm{~km}$ on 28 May at 13:56 LT.


Fig. 8. A multi-blob layer centered at $71 \mathrm{~km}$ on 28 May at 13:10 LT.

lation plot indicates two well-defined peaks at $71.4 \mathrm{~km}$ and $71.75 \mathrm{~km}$.

Figures 6a and $\mathrm{b}$ show sections of the lower altitude layer observed on 27 May in Fig. 4a. This lower altitude structure consists of a thin sheet on the bottom and diffusive blob-like structures above. The layer starts at around $70 \mathrm{~km}$ early in the day and descends to roughly $69 \mathrm{~km}$ by 10:00 LT and then $67.4 \mathrm{~km}$ by 14:00 LT. The SNR map of Fig. 6a shows several small blob-like structures that contribute positive correlations in the form of multiple peaks in the correlation plot. There 



Fig. 9. Thin structures at $71.5,72.8,73.5$, and $74.8 \mathrm{~km}$ on 29 May starting at 10:00, 10:50, 11:12, and 10:40 LT, respectively.
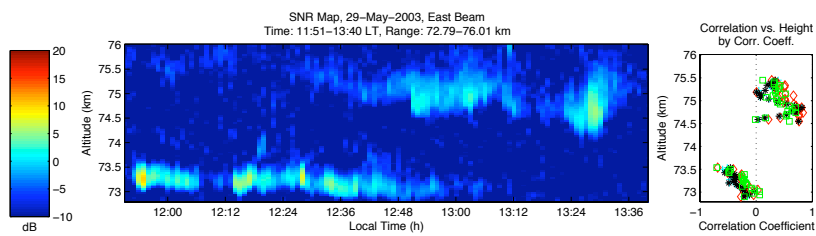

Fig. 10. Two thin structures at 73.2 and $75 \mathrm{~km}$ on 29 May starting at 11:50 and 12:50 LT, respectively.

are two clear peaks at $68.7 \mathrm{~km}$ and $69.25 \mathrm{~km}$ which correspond to the three blobs forming the lower part of the structure at 10:00, 10:06, and 10:15 LT, and the two blobs forming the middle section at 10:11 and 10:21 LT, respectively. The positive correlation above $69.6 \mathrm{~km}$ lacks a strong trend due to the diffuse nature of the structure at those heights, but shows, in general, a decreasing positive correlation in height. The thin sheet below the diffusive structure is more evident in Fig. 6b. The correlation plot shows a narrow peak at $67.4 \mathrm{~km}$. The positive correlation above it is attributed to the diffuse structure above the thin sheet. Again, the diffuse structure produces correlations that lack a clear trend in height, but are in general positive.

Figures 7 and 8 are from 28 May 2003, afternoon data. The correlation plot in Fig. 7 shows dramatically a single peak with negative correlation above and below indicating the presence of a single turbulent layer accompanied by anisotropic edges. The SNR map of Fig. 8 shows a sequence of blobs that developed at roughly $71 \mathrm{~km}$ under the turbulent layer at $73.2 \mathrm{~km}$. The corresponding correlation plot shows both positive and negative correlations with a bias toward positive correlation.

Figures 9 and 10 show SNR maps and correlation plots from 29 May 2003. This day was not as active as the two previous days in terms of large-scale echoing layers. The returns are dominated by thin sheets and short-lived bloblike structures. Two range/time windows were picked that represented the day as a whole.

Figure 9 shows one window with four blob-like structures. Since the structures at $71.5 \mathrm{~km}$ and $74.8 \mathrm{~km}$ are separated in

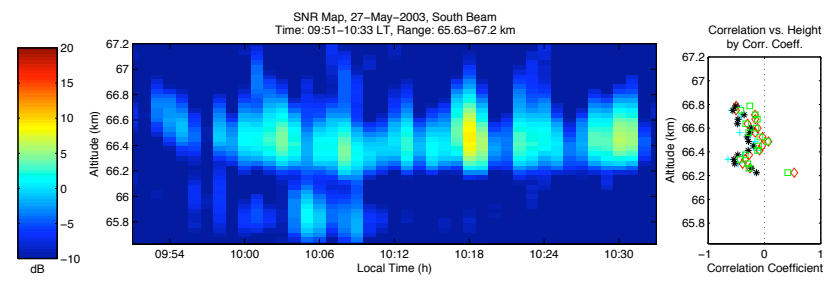

Fig. 11. A thin sheet at $66.5 \mathrm{~km}$ on 27 May at 9:50 LT.

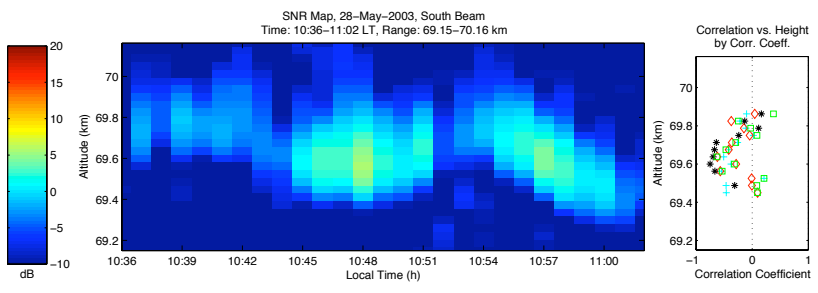

Fig. 12. A thin sheet at $69.6 \mathrm{~km}$ on 28 May at 10:40 LT.

height, they contribute independently to the correlation plot. These topmost and bottommost structures have the familiar single-peaked correlations with decreasing positive correlation above and below the peak. The larger blob-like structure at $73.5 \mathrm{~km}$ and $11: 12 \mathrm{LT}$ and the smaller one at $72.8 \mathrm{~km}$ and 10:50 LT yield positive and negative correlations. It is unclear whether the negative correlation is due to the smaller blob, the larger blob, or a combination of both since the smaller blob occurs right at the lower edge, in altitude, of the larger blob.

Similarly, in Fig. 10 two layers are separated in height to allow for independent contributions to the correlation plot. The top layer forms a single positive peak in the correlation profile, while the bottom layer creates a region of negative correlation. The structures in Figs. 9 and 10 contributing negative correlation are notable since they are two cases of isolated negative correlations. All other cases of negative correlations shown so far have been associated with structures exhibiting positive correlations at neighboring heights, such as those shown in Figs. 5 and 7.

Two other examples of isolated negative correlation from 27 May at $66.5 \mathrm{~km}$, and 28 May 2003, at $69.6 \mathrm{~km}$, are shown in Figs. 11 and 12. These figures are also notable in that they correspond to the only cases of isolated negative correlations found in the entire data set for 27 and 28 May.

Results from 13-14 July 2004, are shown in Figs. 13-16. Only one instance of positive correlation is shown from the two days - see Fig. 13 - since they are similar to positive correlation events observed from May 2003. In general, the turbulent structures above $70 \mathrm{~km}$ showed positive correlation while thin sheets below $70 \mathrm{~km}$ showed negative correlations. Thin sheet examples are shown in Figs. 14-16. 

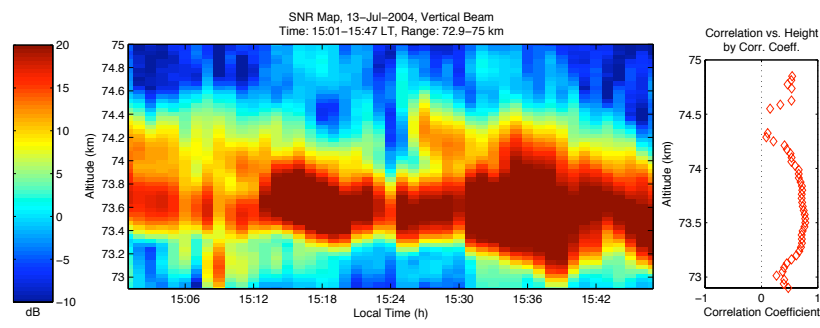

Fig. 13. A turbulent layer at $72.8 \mathrm{~km}$ on 13 July at 15:01 LT.

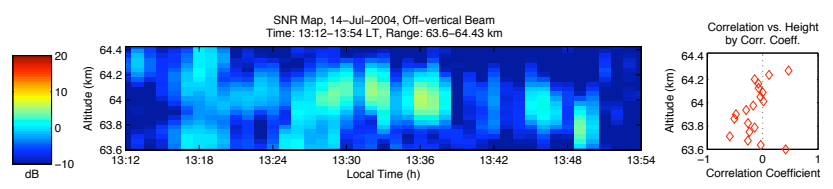

Fig. 14. A thin sheet at $64 \mathrm{~km}$ on 14 July 2004, at 13:15 LT.

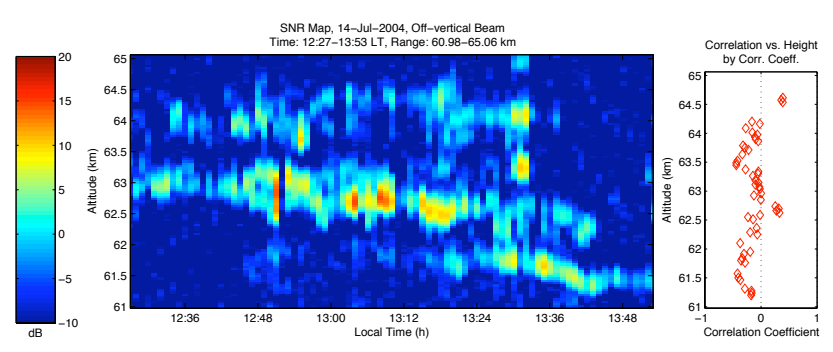

Fig. 15. Thin sheets at $60 \mathrm{~km}$ on 14 July at 12:27 LT.

\section{Discussion}

The full-day correlation plot for 27 May, 2003 - see Fig. 4a - shows mainly positive correlations associated with a strong echoing layer seen to last the entire day at around $74 \mathrm{~km}$ altitude. The matching positive correlation peak at $74 \mathrm{~km}$ can be regarded an indication of turbulence-induced isotropic backscattering mechanism. The slight negative values in the correlation profile on 27 May at higher and lower altitudes are harder to interpret, just as the interpretation of the noisy correlation profile for full-day 29 May (see Fig. 4c) is also difficult.

Our layer-by-layer and event-by-event study of the echoing structures of 27 and 28 May 2003, showed an overwhelming amount of positive correlation events which may be easier to interpret. 29 May 2003 contained a larger number of negative correlation events than the previous two days. Also 13-14 July 2004, showed predominantly positive correlations above $70 \mathrm{~km}$, and cases of negative correlations below $70 \mathrm{~km}$ associated with thin sheet-like echoing structures.

\subsection{Positive correlations of spectral widths and echo power}

In general, positive correlation peaks, ubiquitous throughout the 5 day data set, coincided with the centers of strong

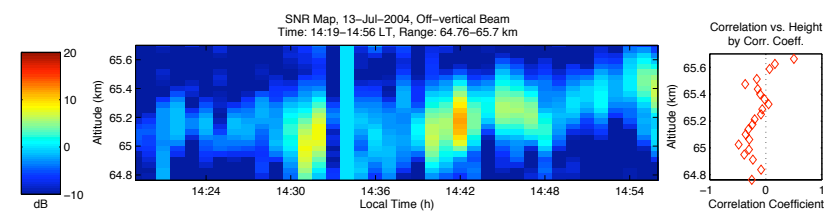

Fig. 16. A thin sheet at $66.2 \mathrm{~km}$ on 13 July at $14: 10 \mathrm{LT}$.

(a)

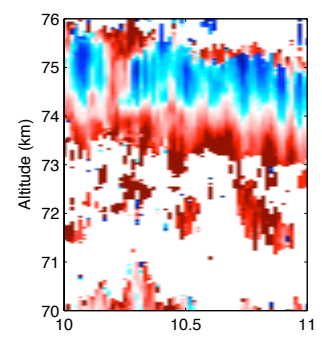

(b)
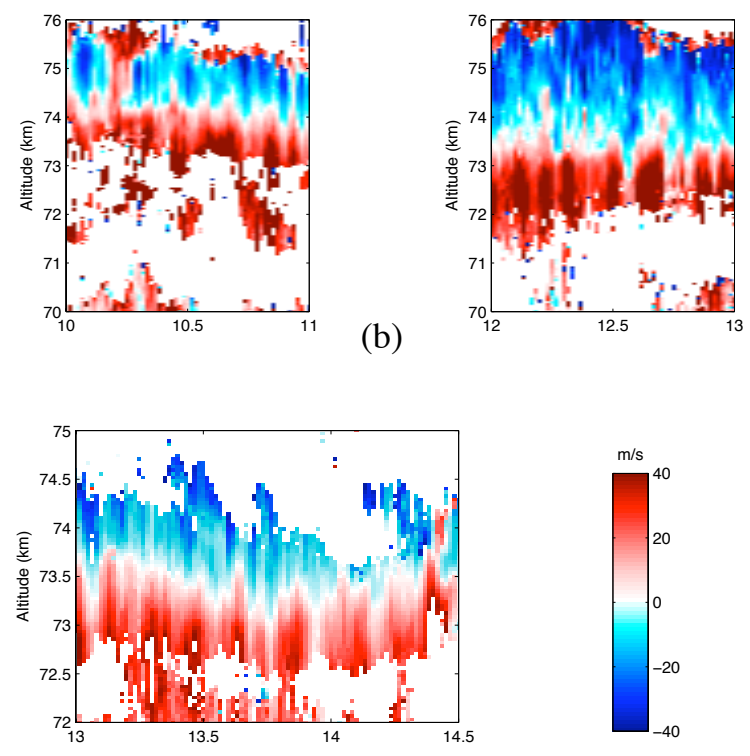

(c)

Fig. 17. Segments of meridional wind maps for 27 May, 2003, (a and b) and 28 May (c).

echoing layers, and the correlations in general decreased towards layer edges, reversing, at times, to negative values at the edge. This signature appears to be consistent with the idea of turbulent layer generation in velocity shear zones as proposed by Bolgiano (1968) and Woodman and Chu (1989). The potential temperature gradient within such layers is expected to be close to zero, with sharp discontinuities at layer edges, and statically stable profiles outside. At the discontinuity it has been postulated that there should be a very thin layer of anisotropic scatterers elongated parallel to the discontinuity (i.e., horizontal in most cases). These anisotropic scatterers should in turn be responsible for aspect sensitive radar echoes with enhanced power for vertical radar beams as compared to off-vertical beams.

It was also proposed by Woodman and Chu (1989) that there should be an enhancement of velocity shear at layer edges. We frequently observed vertical shears in meridional winds co-located with turbulent layers but were unable to resolve or observe the hypothesized enhanced shears at layer edges. Figures $17 \mathrm{a}-\mathrm{c}$ show examples of meridional wind maps from selected periods on 27 and 28 May coinciding in altitude with strong echoing layers. For the large echoing layers of 27-28 May the wind shears lasted the entire day. No 

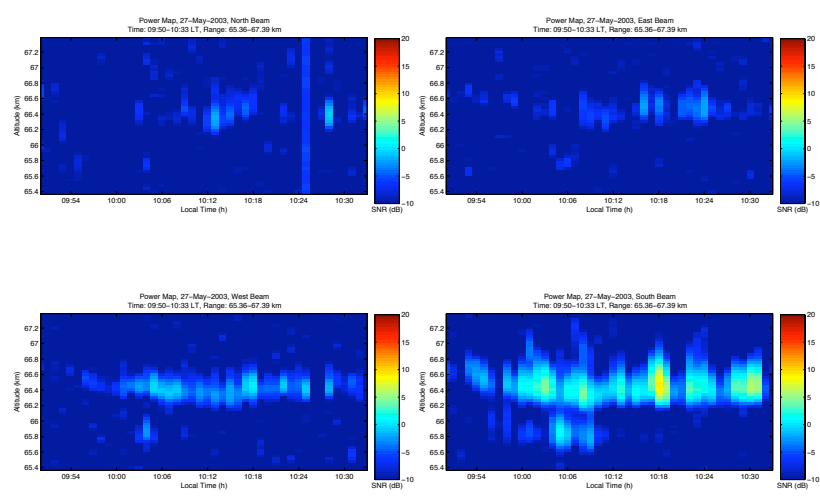

Fig. 18. SNR map in all four beams of event at $66.5 \mathrm{~km}$ on 27 May 2003 at 10:00 LT.

wind shears on the scale of those present during 27-28 May were found on 29 May, which is most likely the reason why backscatter on 29 May was weaker than on the previous two days.

\subsection{Thin sheets and isolated negative correlations}

While negative correlations at layer edges can be accounted for by anisotropic turbulence as discussed above, the cases of isolated negative correlations may require an alternate explanation. As suggested by Fukao et al. (1980) negative correlations could imply radiowave reflections from sharp gradients in electron density. Such gradients, if they persist, would require static stability of the atmosphere (e.g., Gage and Green, 1978) and partial reflected radiowaves from the stable gradients would appear on SNR maps as thin horizontal lines or "sheets". Backscatter from 27-28 May displayed some occurrences of such thin sheets and many more were observed on 29 May. Also, thin sheets were predominant below $70 \mathrm{~km}$ on 13-14 July 2004. The occurrences of isolated negative correlations in our data set were in fact always associated with thin sheets on SNR maps, regardless of the day they occurred.

Figures 11 and 12 display the only two cases of thin sheets with isolated negative correlations observed in 27-28 May. These two cases are potential examples of the partial reflection process just described. However, the sheets in these two events are present in the SNR maps of all four radar beams (EWNS) as shown in Figs. 18 and 19. It is very unlikely that there exist four independent density gradients at different locations at the same altitude with the just the correct tilts so as to cause partial reflected returns into all four radar beams pointing in different directions. Thus anisotropic aspectsensitive scatterers is a more likely explanation than partial reflections for these events. SNR comparisons between the different panels of Figs. 18 and 19 clearly show a beam-tobeam SNR variation which is an indication of aspect sensitivity since the observed SNR differences in the figures are
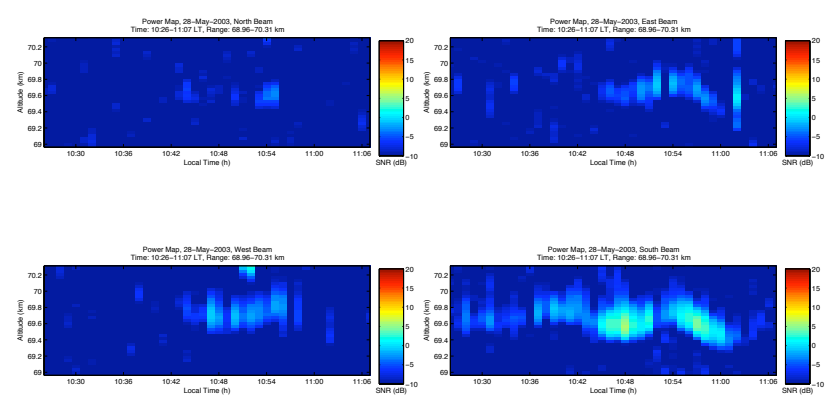

Fig. 19. SNR map in all four beams of event at $69.6 \mathrm{~km}$ on 28 May 2003 at $10: 30$ LT.

well in excess of the channel-to-channel gain differences (of a few dB's in favor of $\mathrm{W}$ and $\mathrm{S}$ channels).

Instead of being composed of pure specular reflectors, it is more likely that thin sheets on SNR maps discussed above correspond to layers of anisotropic scatterers with orientations favorable for strong returns in one beam and weaker returns in others. The layers may even include a mix of isotropic and anisotropic scatterers, with the isotropic scatterers being visible from all viewing directions whereas the anisotropic ones being aspect sensitive. To make sure that the signals received in different beams arrived from independent directions in the sky, we made spectral cross-correlation calculations for the events of Figs. 18 and 19. No significant cross-correlations were found between signals from different beams, which rules out the possibility that the signals were arriving from a single direction in the sky through the sidelobes of the four independent beams.

As further evidence for the independence of different beam signals during events of isolated negative correlations, we next show results from 29 May 2003, during which many instances of isolated negative correlations could be found. Referring to Fig. 9, the short-duration blob-like structure at $73.5 \mathrm{~km}$ and 11:12 LT is shown in Fig. 20 from all four beams along with the corresponding correlation plots (on the top) and spectral width maps (on the bottom). Given the shortness of the event, the correlations were calculated using a minimum spectral width count of five. Also Fig. 21 shows sample spectra from the same event in $2 \mathrm{~min}$ and $75 \mathrm{~m}$ intervals in time and height - the red curves represent the spectral fits of model (5) to measured spectra plotted in blue. Spectra with total SNR of less than $-8 \mathrm{~dB}$ SNR were not fitted and the spectral curves are shown normalized by their peak values to unity. The height of the green arrow represents the relative magnitude of spectral peak to the maximum peak in the data set for that beam.

Clearly, the data shown in Figs. 20 and 21 demonstrate the independence of the signals received by the four radar beams. Furthermore, Fig. 20 shows that the correlation is negative only for the west and south beams - north and east beams had positive correlations between spectral widths and 
echo power. Also, note that west and south beams have, on the average, narrower spectra than found in the north beam.

These observations suggest a combination of aspectsensitive and isotropic scatterers for the event. Another example of an aspect-sensitive structure from 29 May exhibiting a mix of positive and negative correlations is shown in Fig. 22 which gives further information about a layer first shown in Fig. 10. Positive correlations are observed in the north and west beams whereas the remaining two beams show a mix of positive and negative correlations. Again, it is most likely that the echoing sheet is composed of anisotropic scatterers rather than pure specular reflectors.

Many of the thin sheets analyzed from 29 May that showed isolated negative correlation in one beam actually showed mixed cases of correlation when viewed through all four beams. Isolated negative correlations never occurred in all four beams and the observed aspect sensitivity was similar to the structures in Figs. 20 and 22 which showed strong returns in one or two beams and weak returns in the others.

\subsection{Vertical beam data and aspect sensitivity}

A large fraction of the data examined and presented in this study were collected with oblique radar beams. This is in contrast to earlier studies of Fukao et al. (1980) and Røyrvik (1983) conducted with vertical beams. These earlier studies showed a significant amount of negative correlations in the lower mesosphere below $75 \mathrm{~km}$. The oblique beam data from May 2003 examined here do not show as strong an evidence of separate altitude regimes for positive and negative correlation, but the data from July 2004, which included vertical beam observations, show an abundance of thin sheets from primarily below $70 \mathrm{~km}$ with associated negative correlations in agreement with Fukao et al. (1980) and Røyrvik (1983).

However, vertical beam and oblique beam comparisons from July 2004 data show that thin sheets are consistently detected in both beams, which once again argues against the idea of pure specular reflections, and strengthens the evidence obtained from the oblique beam data that thin sheets detected primarily in the lower mesosphere probably consist of a mix of isotropic and aspect sensitive scatterers.

\subsection{Summary}

Overall, all multi-beam experiments, like our May 2003 and July 2004 observations, seem to produce backscattered SNR maps from the mesosphere which are highly correlated from beam to beam down to their minute details; dissimilar features from beam to beam correspond to a negligible fraction of the overall received power in all cases examined. This fact, in itself, constitutes a strong evidence that pure specular reflections play a negligible role, if any, in causing mesospheric VHF radar echoes. Furthermore, what is detected invariably in all beams but often to varying strengths indicative of aspect sensitivity - seems to never exhibit exclusively
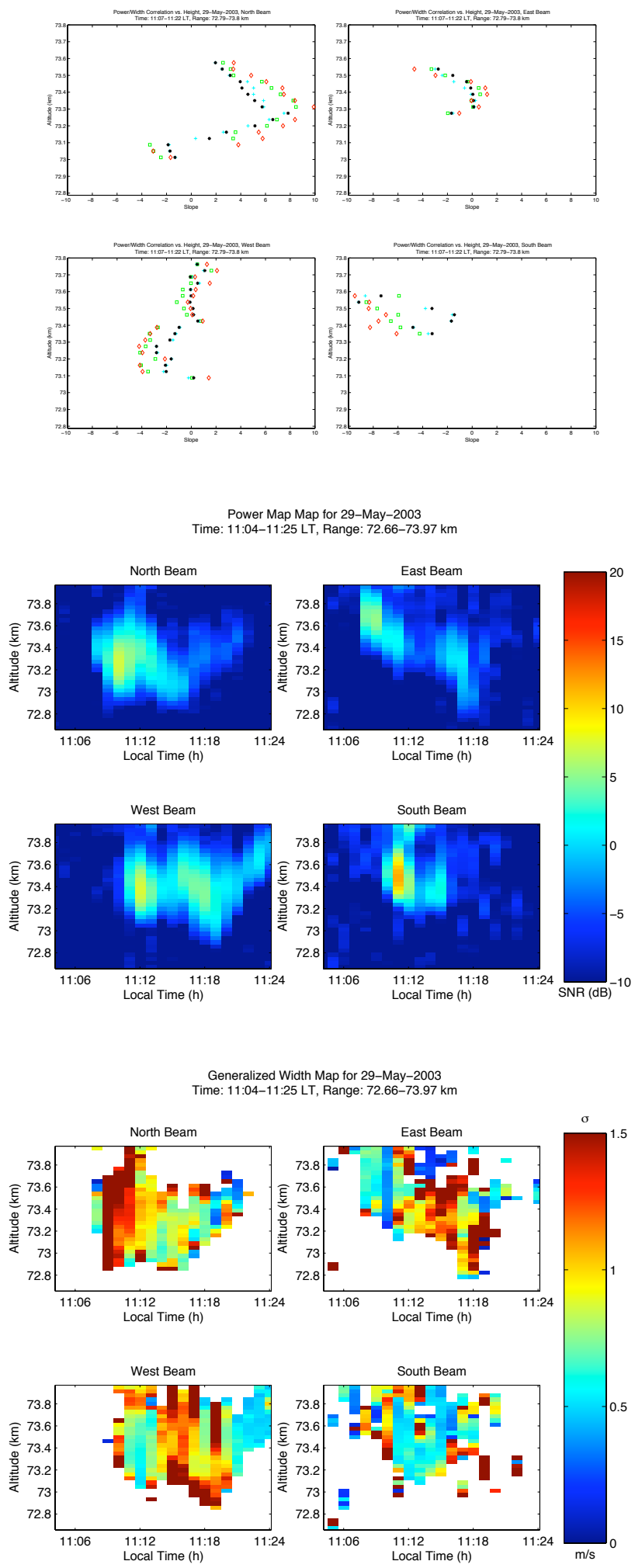

Fig. 20. SNR map and spectral width map of event at $73.4 \mathrm{~km}$ on 29 May 2003 at 11:10 LT. 

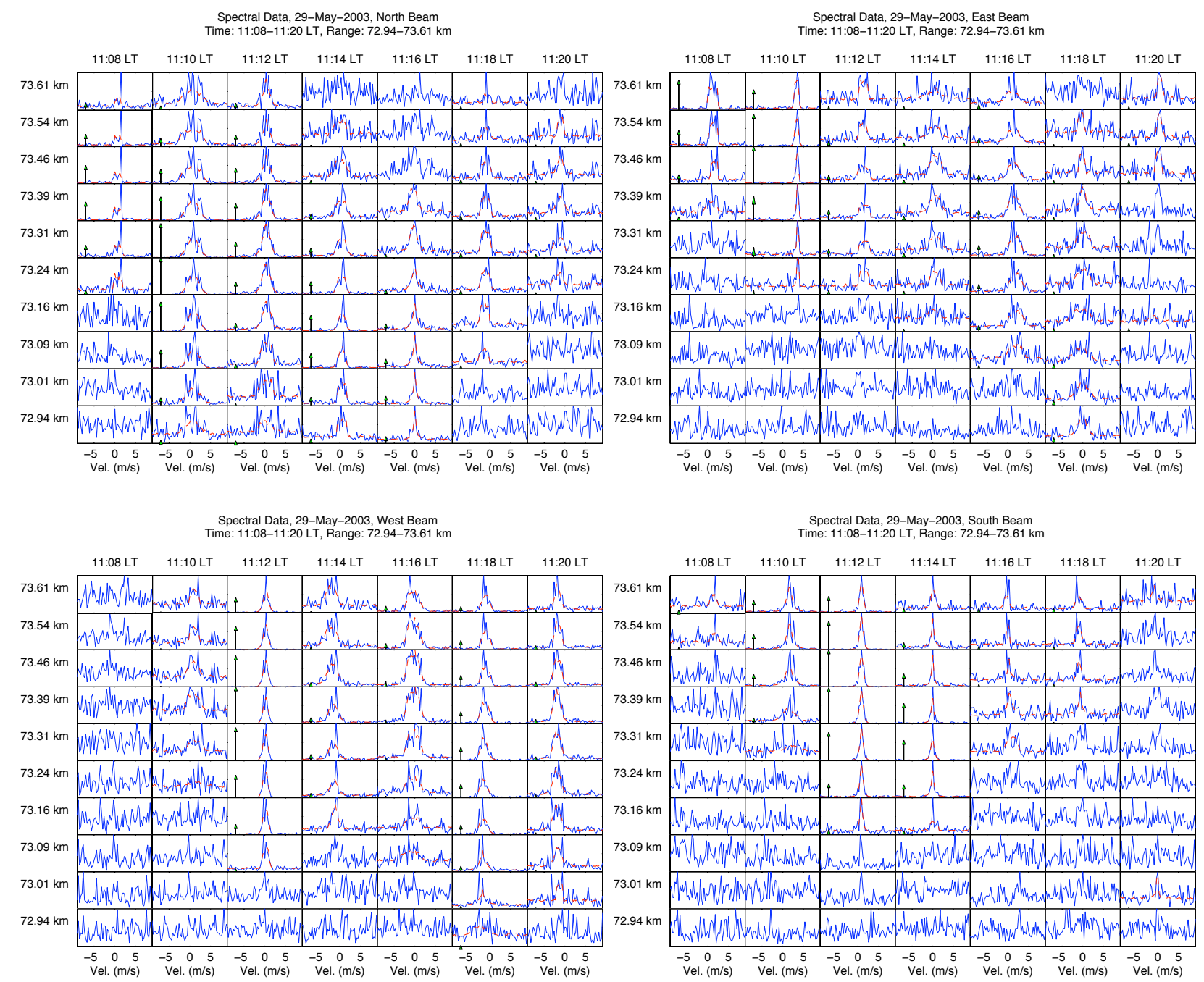

Fig. 21. Spectral data and curve fits from all four beams of event at $73.4 \mathrm{~km}$ on 29 May 2003 at 11:10 LT.
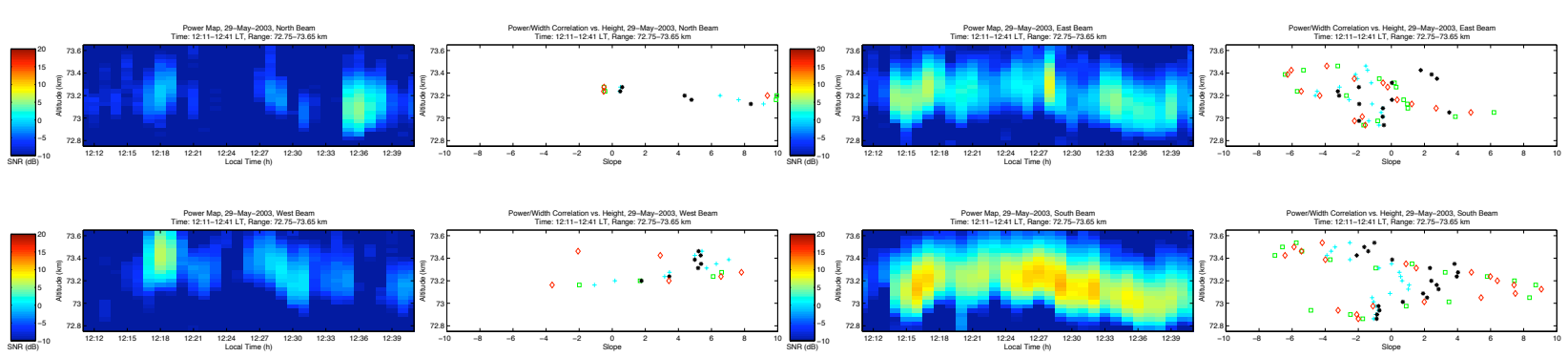

Fig. 22. SNR map and correlation plots of all four beams of event at $73.25 \mathrm{~km}$ on 29 May 2003 at 12:11 LT.

negative correlations between spectral widths and the echo power in all the radar beams. In view of these facts, turbulent and partially aspect-sensitive backscatter remains to be the most likely mechanism responsible for VHF radar echoes from the mesosphere.
Perhaps the most significant altitudinal trends implied by all the past and present data sets are (i) the decrease of scattering layer widths with decreasing altitude on most days, and (ii) the increase in aspect sensitivity with decreasing height. These two trends may even be self-consistent if, as 
a rule, all scattering layers are more aspect-sensitive at the edges as compared to their centers, consistent with ideas of Bolgiano (1968) and others referenced earlier - thin layers will have a larger surface area to volume ratio and thus appear more aspect sensitive than thicker layers, in particular if the radar pulse is unable to resolve the layers. As for trend (i), decreasing layer width with decreasing altitude, it seems to be a straightforward consequence of the height variation of turbulence outer scales determined by gravity wave filtering throughout the mesosphere (e.g., Fritts and Alexander, 2003).

\section{Conclusions}

Our extensive and high-resolution Jicamarca based study of mesospheric echoing events detected by vertical as well as oblique radar beams failed to produce any strong evidence for specular reflections playing a substantial role in the production of mesospheric VHF radar returns. The data was more consistent with backscatter from turbulent layers, which in turn exhibited varying degrees of anisotropy/aspectsensitivity signatures depending on altitude, background conditions, as well as scattering altitudes within the turbulent layers when they could be resolved.

The correlation profiles shown in this paper between spectral widths and echo power give experimental evidence to the idea that VHF backscatter from turbulent layer edges should exhibit stronger aspect sensitivities because of a combination of dynamic and geometrical considerations (e.g., Bolgiano, 1968; Woodman and Chu, 1989). However, a higher reflectivity and enhanced shears predicted for the layer edges by unstable shear layer simulations described in Gibson-Wilde et al. (2000) was not seen and/or resolved. Further studies will be needed to determine the role of velocity shears and whether dynamic or convective processes dominate the generation of the layers observed by the radar.

Acknowledgements. We thank the staff and engineers of the Jicamarca Radio Observatory for their assistance with the observations. Jicamarca Observatory is operated by the Instituto Geofisico del Peru, with support from the National Science Foundation. This work was supported by the Aeronomy Program, Division of Atmospheric Sciences of the NSF through grants ATM 04-22661 and 04-22837.

Topical Editor U.-P. Hoppe thanks D. A. Holdsworth and O. Royrvik for their help in evaluating this paper.

\section{References}

Bahcivan, H., Hysell, D. L. and Kelley, M. C.: Phase diffusion and random walk interpretations of electromagnetic scattering, Phys. Rev. E, 68, 021101, 2003.

Bolgiano Jr., R.: The general theory of turbulence: Turbulence in the atmosphere, in: Wind and Turbulence in the Stratosphere, Mesosphere, and Ionosphere, edited by: Rawer, K., northHolland, New York, 371-400, 1968.
Czechowsky P. and Rüster, R.: VHF radar observations of turbulent structures in the polar mesopause region, Ann. Geophys., 15, 1028-1036, 1997.

Fritts, D. C. and Alexander, J.: Gravity wave dynamics and effects in the middle atmosphere, Rev. Geophys., 41, 1, doi:10.1029/2001RG0001106, 2003.

Fukao, S., T. Sato, Harper, R. M., and Kato, S.: Radio wave scattering from the tropical mesosphere observed with the Jicamarca radar, Radio Sci., 15, 447-457, 1980.

Gage, K. S. and Green, J. L.: Evidence for specular reflection from monostatic VHF radar observations of the stratosphere, Radio Sci., 13, 991-1001, 1978.

Gage, K. S. and Balsley, B. B.: On the scattering and reflection mechanisms contributing to clear air radar echoes from the troposphere, stratosphere, and mesosphere, Radio Sci., 15, 243-257, 1980.

Gibson-Wilde, D., Werne, J., Fritts, D., and Hill, R.: Direct numerical simulations of VHF radar measurements of turbulence in the mesosphere, Radio Sci., 35, 783-798, 2000.

Hocking, W. K. and Röttger, J.: The structure of turbulence in the middle and lower atmosphere seen by and deduced from MF, HF, and VHF radar, with special emphasis on small-scale features and anisotropy, Ann. Geophys., 19, 933-944, 2001.

Hocking, W. K., Fukao, S., Tsuda, T., Yamamoto, M., Sato, T., and Kato, S.: Aspect sensitivity of stratospheric VHF radio wave scatterers , particularly above 15-km altitude, Radio Sci., 25, 613-627, 1990.

Kudeki, E.: Radar interferometer observations of mesospheric echoing layers at Jicamarca, J. Geophys. Res., 93, 5413-5421, 1988.

Kudeki, E., Bhattacharyya, S., and Woodman, R. F.: A new approach in incoherent scatter $\mathrm{F}$ region $\mathrm{E} \times \mathrm{B}$ drift measurements at Jicamarca, J. Geophys. Res., 104, 28 145-28 162, 1999.

Reid, I. M.: Radar observations of stratified layers in the mesosphere and lower thermosphere (50-100 km), Adv. Space Res., 10, 7-19, 1990.

Röttger, J. and C. H. Liu, Partial reflection and scattering of VHF radar signals from the clear atmosphere, Geophys. Res. Lett., 5, 357-360, 1978.

Röttger, J., Rastogi, P. K., and Woodman, R. F.: High-resolution VHF radar observations of turbulence structures in the mesosphere, Geophys. Res. Lett., 6, 617-620, 1979.

Røyrvik, O.: VHF radar signals scattered from the equatorial mesosphere, Radio Sci., 18, 1325-1335, 1983.

Røyrvik, O.: Relationship between scattered power and correlation time in VHF radar signals, Radio Sci., 20, 212-220, 1985.

Woodman, R. F. and Chu, Y.-H.: Aspect sensitivity measurements of VHF backscatter made with the Chung-Li radar: Plausible mechanisms, Radio Sci., 24, 113-125, 1989.

Yamamoto, M., Sato, T., May, P. T., Tsuda, T., Fukao, S., and Kato, S.: Estimation error of spectral parameters of mesospherestratosphere-troposphere radars obtained by least squares fitting method and its lower bound, Radio Sci., 23, 1013-1021, 1988. 